\title{
UM PANORAMA SOBRE AVALIAÇÃO DE CICLO DE VIDA COM BASE NOS ANAIS DO SIMPÓSIO DE ENGENHARIA DE PRODUÇÃO
}

\section{A LIFE CYCLE ASSESSMENT PERSPECTIVE CONSIDERING THE PRODUCTION ENGINEERING SYMPOSIA PROCEEDINGS}

\author{
Camila Daniele Willers ${ }^{1}$; Luciano Brito Rodrigues ${ }^{2}$ \\ ${ }^{1}$ Universidade Estadual do Sudoeste da Bahia - UESB - Itapetinga - Brasil \\ camiladw@hotmail.com \\ ${ }^{2}$ Universidade Estadual do Sudoeste da Bahia - UESB - Itapetinga - Brasil \\ rodrigueslb@gmail.com
}

\begin{abstract}
Resumo
Nos últimos anos a Avaliação de Ciclo de Vida (ACV) vem ganhando maior atenção no Brasil, com sua difusão nos principais centros de pesquisa do país. Apesar de recente, faz-se necessário conhecer como andam os desenvolvimentos sobre o tema no país, o que pode ser feito por meio da verificação da produção científica nacional. Assim, neste trabalho se realizou uma pesquisa bibliográfica sobre a ACV nos anais do Simpósio de Engenharia de Produção - SIMPEP, para seleção dos artigos que abordaram o respectivo tema. Em seguida, foi aplicada uma pesquisa exploratória e descritiva nos trabalhos selecionados, identificando as Instituições de Pesquisa, os temas relacionados à ACV e os estudos de casos que aplicaram a metodologia. Por último, foram analisados somente os trabalhos que aplicaram a ACV estudos de casos, nos quais foram identificados aspectos mais específicos para uma comparação entre os mesmos. A pesquisa selecionou 39 artigos, e constatou a participação de 30 diferentes instituições pesquisa, sendo 27 destas universidades brasileiras. Ressalta-se ainda que dos 39 artigos selecionados, 35 se restringiram a uma abordagem conceitual da ACV, sem uma aplicação efetiva da metodologia, cujas citações estavam principalmente relacionadas com o Sistema de Gestão Ambiental $e$ Sustentabilidade. Assim, foi encontrado um baixo número de trabalhos, apenas 10,3\%, que aplicaram efetivamente a metodologia da ACV. Verifica-se que destes trabalhos, três foram publicados em edições recentes do evento (2007, 2008 e 2009), refletindo a recente tendência de trabalhos mais consistentes sobre ACV no SIMPEP.
\end{abstract}

Palavras-chave: Avaliação de Ciclo de Vida; NBR ISO 14040; pesquisa bibliográfica.

\section{Introdução}

O impacto das atividades humanas sobre o meio ambiente não é um fenômeno recente. Historicamente tem-se observado um desencadeamento de fatos contribuintes e agravantes da degradação ambiental vivenciada globalmente, que vão desde o advento do desenvolvimento das atividades agrícolas, passando pela Revolução Industrial, até culminar no atual modo de vida 
capitalista. Paralelo a este cenário, a preocupação ambiental surge como pauta de discussões, em termos mundiais, somente em tempos recentes (BORGES; TACHIBANA, 2005). Nesse contexto, torna-se importante a utilização de instrumentos que analisem a contribuição de uma tecnologia para a sustentabilidade e avaliem seus impactos ambientais (FIGUEIRÊDO et al., 2007). A resposta surge em forma de novas técnicas, as quais visam mitigar os impactos, e que podem ser soluções únicas ou um conjunto destas. Como exemplo, pode-se citar o Sistema de Gestão Ambiental, a Rotulagem Ambiental, a Avaliação de Desempenho Ambiental, a Auditoria Ambiental, a Avaliação de Ciclo de Vida, a Produção Mais Limpa, o Ecodesign, entre outras.

A Avaliação de Ciclo de Vida (ACV) é uma metodologia de gestão ambiental que estabelece uma visão geral das conseqüências ambientais da existência de um produto/processo. Ela engloba o ciclo de vida do produto, desde a extração das matérias-primas, envolvendo sua produção e uso, as possibilidades de reciclagem e reuso, até sua disposição final (SANTOS, 2006). Por isso a ACV é denominada de metodologia do "berço ao túmulo", e tem como vantagens poder revelar os potenciais, mas nem sempre evidentes impactos ambientais (SILVA et al., 2009). A ACV é uma ferramenta para múltiplos usos, como subsídio às estratégias de marketing (como declarações ambientais ou rotulagens), na tomada de decisão, na melhoria ambiental do produto, na concepção do projeto, na avaliação ambiental de processos e na definição políticas ambientais. Esta ferramenta é vantajosa para produtores e empresas poderem quantificar e comparar de uma forma integrada a performance ambiental relativa de seus produtos, e estabelecerem ações proativas as pressões ambientais dos mercados (CHEHEBE, 1997; SANTOS, 2006).

Uma vez que os resultados produzidos pela área acadêmica são divulgados preferencialmente na forma de artigos científicos em conferências e periódicos, a hipótese de pesquisa deste trabalho considerou investigar o progresso dos estudos sobre avaliação de ciclo de vida desenvolvidos no Brasil, a partir de uma pesquisa nos Anais do Simpósio de Engenharia de Produção (SIMPEP). A escolha se deve ao fato do evento possuir expressão nacional, sendo uma das principais formas para a divulgação de trabalhos da área da Engenharia de Produção, na qual o tema avaliação de ciclo de vida está inserido, e também em virtude da facilidade de acesso ao material de pesquisa, visto que estes estão disponíveis gratuitamente na internet. Ressalta-se que existem outras bases nacionais com as mesmas características da escolhida. Entretanto, a apresentação e devida discussão dos resultados dos trabalhos a serem encontrados em todas possíveis bases não se adequariam ao escopo deste artigo, que propõe uma discussão mais aprofundada, com fins de conhecer o panorama dos trabalhos e das metodologias aplicadas pelos autores para realização de uma ACV.

$\mathrm{O}$ artigo primeiro apresenta o estado da arte da metodologia de ACV, seguindo de uma pesquisa documental, para seleção dos artigos que abordaram o respectivo tema. Em seguida é 
aplicada uma pesquisa exploratória e descritiva nos trabalhos selecionados, identificando as Instituições de Pesquisa, os temas relacionados à ACV e os estudos de casos que aplicaram a metodologia. Posteriormente, são abordados somente os estudos de casos que utilizaram a ACV, nos quais são identificados os campos de aplicação da ACV no país, as metodologias empregadas, os programas computacionais utilizados e os benefícios e limitações da ACV. Por fim, o texto traz as considerações sobre o trabalho, com conclusões e sugestões para maior divulgação dos estudos sobre ACV.

\section{Revisão bibliográfica}

\subsection{História da ACV}

Os princípios da Avaliação de Ciclo de Vida foram desenvolvidos nos Estados Unidos no final da década de 1960, quando preocupações com as limitações das matérias-primas e recursos energéticos despertaram o interesse em encontrar maneiras quantificar o uso de energia e os recursos para projetos futuros e sua utilização, devido à crise energética deflagrada pelo aumento do preço do petróleo (SANTOS, 2006; VIGON et al., 1993). Um dos primeiros estudos quantificando as necessidades de recursos, emissões e resíduos originados por diferentes embalagens de refrigerantes foi conduzido pelo Midwest Research Institute (MRI) para a Companhia Coca Cola em 1969. Este processo de quantificação da utilização de recursos naturais e dos índices de emissão tornou-se conhecido como Resource and Environmental Profile Analysis (REPA) (HUNT; FRANKLIN, 1996; CHEHEBE, 1997). Esse modelo foi aprimorado em 1974 pelo MRI, durante a realização de um estudo para a EPA (Environmental Protection Agency) sobre embalagens de cervejas, e é muitas vezes referenciado como um marco para o surgimento do que hoje conceituamos como ACV (GUINÉE, 1995; CHEHEBE, 1997). Este estudo foi de longe o mais ambicioso até o referido momento, pois, envolveu a indústria do vidro, aço, alumínio, papel e plástico e todos os fornecedores daquelas indústrias, tendo-se caracterizado mais de 40 materiais (HUNT; FRANKLIN, 1996). Na Europa, foi também desenvolvido um procedimento similar chamado de Ecobalance (VIGON et al., 1993).

De 1975 até o início de 1980 o interesse por estes estudos abrangentes diminuiu por causa da influência do fim da crise do petróleo, e a preocupação ambiental passou a questões de gestão de resíduos perigosos (VIGON et al., 1993). Em 1988, quando os resíduos sólidos se tornaram um problema a nível mundial, a ACV novamente surgiu como uma importante metodologia para analisar os problemas ambientais (VIGON et al., 1993). Então, no final da década de 80 e toda a década de 90 foram pautados pelo esforço internacional para normalizar os princípios e técnicas da ACV e para desenvolver procedimentos de boa conduta (SANTOS, 2006). A normalização da ACV começou na International Organization for Standardization (ISO), que publicou a série ISO 14000, 
na qual engloba a ACV nas normas:

- ISO 14040. Life Cycle Assessment. Principles and Framework, (2006): Avaliação do Ciclo de Vida. Princípios e Estrutura;

- ISO 14044. Life Cycle Assessment. Requirements and Guidelines, (2006): Avaliação do Ciclo de Vida. Requisitos e Orientações;

- ISO/TR 14047. Life Cycle Impact Assessment. Examples of Application of ISO 14042, (2000): Avaliação de Impacto do Ciclo de Vida. Exemplos de Aplicação da ISO 14042;

- ISO/TS 14048. Life Cycle Assessment. Data Documentation Format, (2001): Avaliação do Ciclo de Vida. Formato de dados e documentação;

- ISO/TR 14049. Life Cycle Assessment. Examples of Application of ISO 14041 for goal and scope definition and inventory analysis, (2000): Avaliação do Ciclo de Vida. Exemplos de Aplicação da ISO 14041 para definição do objetivo e do escopo e análise de inventário.

\subsection{Panorama da ACV no Brasil}

No Brasil, a história da ACV inicia na década de 90, quando foi criado o Subcomitê de ACV da Associação Brasileira de Normas Técnicas, que passou incorporar o Comitê Técnico 207 da ISO, através do Grupo de Apoio à Normalização Ambiental (Gana). Em 1997, foi lançado o primeiro livro nacional que tratou sobre esta temática, “Análise do Ciclo de Vida de Produtos - Ferramenta Gerencial da ISO 14040”, de José Ribamar B. Chehebe. No período de 1997 a 1999, foi desenvolvido o primeiro estudo brasileiro denominado "Análise do Ciclo de Vida de embalagens para o mercado brasileiro", realizado pelo Centro de Tecnologia de Embalagem (Cetea), do Instituto de Tecnologia de Alimentos (Ital). Já em 2002, foi criada a Associação Brasileira de Ciclo de Vida (ABCV), que obteve destaque na coordenação da segunda Conferência Internacional de Avaliação de Ciclo de Vida (CILCA), realizada na cidade de São Paulo, em 2007, consolidando o tema ACV no Brasil. Também, destaca-se a criação do Projeto Brasileiro de Inventário do Ciclo de Vida para a Competitividade da Indústria Brasileira, do Instituto Brasileiro de Informação em Ciência e Tecnologia (IBICT), que desde 2002 vem atuando para o desenvolvimento do Inventário do Ciclo de Vida nacional (LIMA, 2007).

Quanto à aplicação da ACV no meio empresarial brasileiro, uma pesquisa desenvolvida por Lima (2007) identificou que sete empresas que haviam aplicado a ACV no Brasil, sendo duas nacionais e as demais multinacionais com origem européia. A autora destaca que isso demonstra a importância deste continente para o desenvolvimento da ACV, e que entre essas sete empresas, os estudos desenvolvidos estão associados a uma estratégia de mercado, devido à imagem que seus produtos representam para a sociedade. 


\subsection{A metodologia de Avaliação de Ciclo de Vida (ACV)}

Os princípios e técnicas para realização de uma ACV são normalizados pela ISO 14040 (2006), que conceitua a ACV como uma compilação e avaliação das entradas, das saídas e dos impactos ambientais potenciais de um sistema de produto ao longo do seu ciclo de vida. A norma divide a realização da ACV em quatro fases (Figura 1).

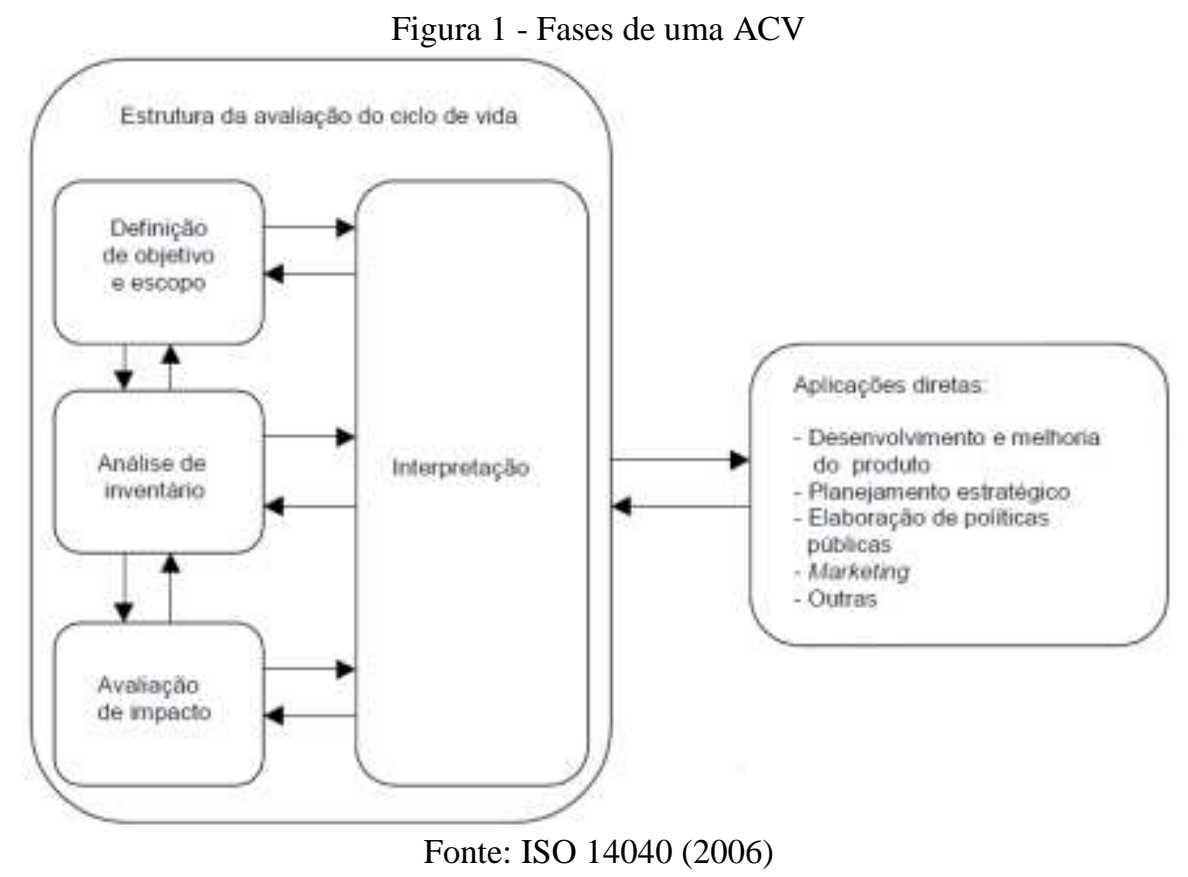

- Objetivo e Escopo: o objetivo de um estudo da ACV deve declarar inequivocamente a aplicação pretendida, as razões para conduzir o estudo e o público-alvo. Na definição do escopo devem ser considerados e claramente descritos os seguintes itens: a) Função e unidade funcional; b) Fronteiras do sistema; c) Requisitos da qualidade dos dados; d) Comparações entre sistemas; e) Considerações sobre análise crítica;

- Análise de inventário: envolve a coleta de dados e procedimentos de cálculo para quantificar as entradas e saídas pertinentes de um sistema de produto. Estes dados também constituem a entrada para a avaliação do impacto do ciclo de vida;

- Avaliação de impactos: é dirigida à avaliação da significância de impactos ambientais potenciais, usando os resultados da análise de inventário do ciclo de vida. Em geral, este processo envolve a associação de dados de inventário com impactos ambientais específicos e a tentativa de compreender estes impactos;

- Interpretação de resultados: a fase da ACV na qual as constatações da análise do inventário e da avaliação de impacto ou, no caso de estudos de inventário do ciclo de vida, somente os resultados da análise de inventário, são combinados, de forma consistente, com o objetivo e o escopo definidos, visando alcançar conclusões e recomendações. 
A ACV pode ser realizada de modo parcial e ainda assim obter resultados significativos. Por exemplo, segundo Vigon et al. (1993), os resultados do inventário podem fornecer uma direção para realização de esforços para mudanças, mostrando quais etapas requerem mais energia ou outros recursos, ou quais etapas contribuem com a maioria dos poluentes. Esta aplicação é especialmente relevante para estudos internos para dar suporte nas decisões de prevenção à poluição, conservação de recursos, e oportunidades de redução na geração de resíduos.

Já a avaliação de impacto identifica as alterações ecológicas, as conseqüências na saúde humana e o esgotamento dos recursos naturais, bem como outros efeitos, tais como alteração do habitat, que não podem ser analisados no inventário. Sendo que a coleta e definição de dados dão suporte à etapa de avaliação de impacto que pode ocorrer como parte da preparação do inventário. Assim, as etapas da ACV podem ser realizadas separadas, mas seus componentes estão interrelacionados (VIGON et al., 1993).

No entanto, ainda é válido destacar que a metodologia de ACV pode se diferenciar nos estudos principalmente na fase de Avaliação de Impacto, que de acordo com a ISO 14044, é um processo qualitativo e/ou quantitativo. Sendo assim, pode-se dividir os estudos, rotulando as ACV's qualitativas ou semi-quantitativas como ACV's Simplificadas, que são normalmente apresentadas em forma de questionários cobrindo diferentes critérios selecionados e em matrizes (UFSC, 2011). Quanto as ACV's quantitativas, segundo Pegoraro (2008), estas também podem ser dividas em dois principais tipos de métodos a serem desenvolvidos para a fase de Avaliação do Impacto do Ciclo de Vida, que são os (i) Métodos clássicos de Avaliação de Impacto e os (ii) Métodos de Danos Orientados.

\section{Material e método da pesquisa}

Como técnica de coleta dados, utilizou-se a pesquisa documental, tendo como fonte de dados os anais do Simpósio de Engenharia de Produção (SIMPEP), de 1999 a 2010, disponíveis na página do evento no endereço http://www.simpep.feb.unesp.br/anais.php. O caráter da escolha se deve ao fato do evento possuir expressão nacional, sendo uma das principais formas para a divulgação de trabalhos da área da Engenharia de Produção, na qual o tema avaliação de ciclo de vida está inserido, e também em virtude da facilidade de acesso ao material de pesquisa, visto que estes estão disponíveis gratuitamente na internet. A importância do Evento se confirma através dos números divulgados em sua última edição, na qual, teve 723 trabalhos inscritos, com 950 participantes e dentre as sessões temáticas, foi verificado 58 sub-temas. Pode-se verificar a amplitude do Evento, tanto em relação ao número de participantes, quanto à abordagem dos mais diversos temas e pela inovação destes. Além disso, ressalta-seque o SIMPEP obteve a sua qualificação junto à Coordenação de Aperfeiçoamento de Pessoal de Nível Superior, que apóia a 
realização deste evento científico juntamente com o Conselho Nacional de Desenvolvimento Científico e Tecnológico e a Fundação de Amparo à Pesquisa do Estado de São Paulo.

A busca nos anais do SIMPEP se realizou em forma de palavras-chave, fundamentando a escolha destas em assuntos que poderiam estar relacionados direta e indiretamente com a ACV, e nas questões semânticas dos idiomas estrangeiros, inglês e espanhol, que também são aceitos pelo Evento. As palavras utilizadas foram: life cycle, ACV, ciclo de vida, ISO 14040, ecodesign, $\mathrm{P}+\mathrm{L}$, produção mais limpa e produção limpa. Nos primeiros resultados obtidos foi realizado um processo de seleção, devido ao volume de literatura selecionado pela pesquisa, para identificar apenas os trabalhos que citavam a ACV em seu contexto, alcançando assim o objetivo desta pesquisa.

Após a seleção dos artigos, a pesquisa se caracterizou por ser exploratória e descritiva. Segundo Gil (1995), exploratória porque é realizada com o objetivo de desenvolver, esclarecer e modificar conceitos e idéias; e descritiva por ter como objetivo a descrição das características do objeto em estudo ou fenômeno ou o estabelecimento de relações entre variáveis. Deste modo, a pesquisa buscou identificar as Instituições de Pesquisa, os temas relacionados à ACV e os estudos de casos que aplicaram a metodologia. Em uma terceira etapa, foram analisados, através de uma investigação empírica, os estudos de casos que aplicaram a metodologia de ACV, onde foram abordados itens definidos como os campos de aplicação da ACV no país, as metodologias empregadas, os programas computacionais utilizados e os benefícios e limitações da ACV.

\section{Análise de dados e resultados}

A pesquisa identificou primeiramente 39 artigos que citavam ACV. A leitura destes permitiu classificá-los nas quatro categorias definidas pela pesquisa, abordados separadamente nos tópicos a seguir:

- Instituições de pesquisa;

- Áreas de atuação;

- Metodologia empregada na aplicação da ACV; e

- Benefícios e limitações identificadas na metodologia de ACV.

As duas últimas categorias serão discutidas somente com as publicações que aplicaram a metodologia da ACV em estudo de caso.

\subsection{Instituições de pesquisa}

Esta categoria identificou as instituições de pesquisa que realizam investigações na área da $\mathrm{ACV}$, ou mesmo que abordaram o assunto indiretamente em suas pesquisas. Foram encontrados 39 artigos, contabilizando a participação de 30 diferentes instituições, sendo 27 universidades 
brasileiras, 1 universidade portuguesa e 2 empresas. Em relação à freqüência (o número de vezes que cada instituição de pesquisa foi encontrada em diferentes artigos), foram identificadas as universidades relacionadas na figura 2 como as principais instituições de pesquisa sobre ACV, observando que para elaboração da figura foram consideradas apenas aquelas com frequiência superior a um.

Figura 2 - Freqüência das instituições de pesquisa (número de vezes que instituições de pesquisa foram encontradas em diferentes artigos) que abordaram o tema Avaliação de Ciclo de Vida (ACV). Pesquisa realizada nos anais do Simpósio de Engenharia de Produção (SIMPEP), no período de 1999 a 2010.

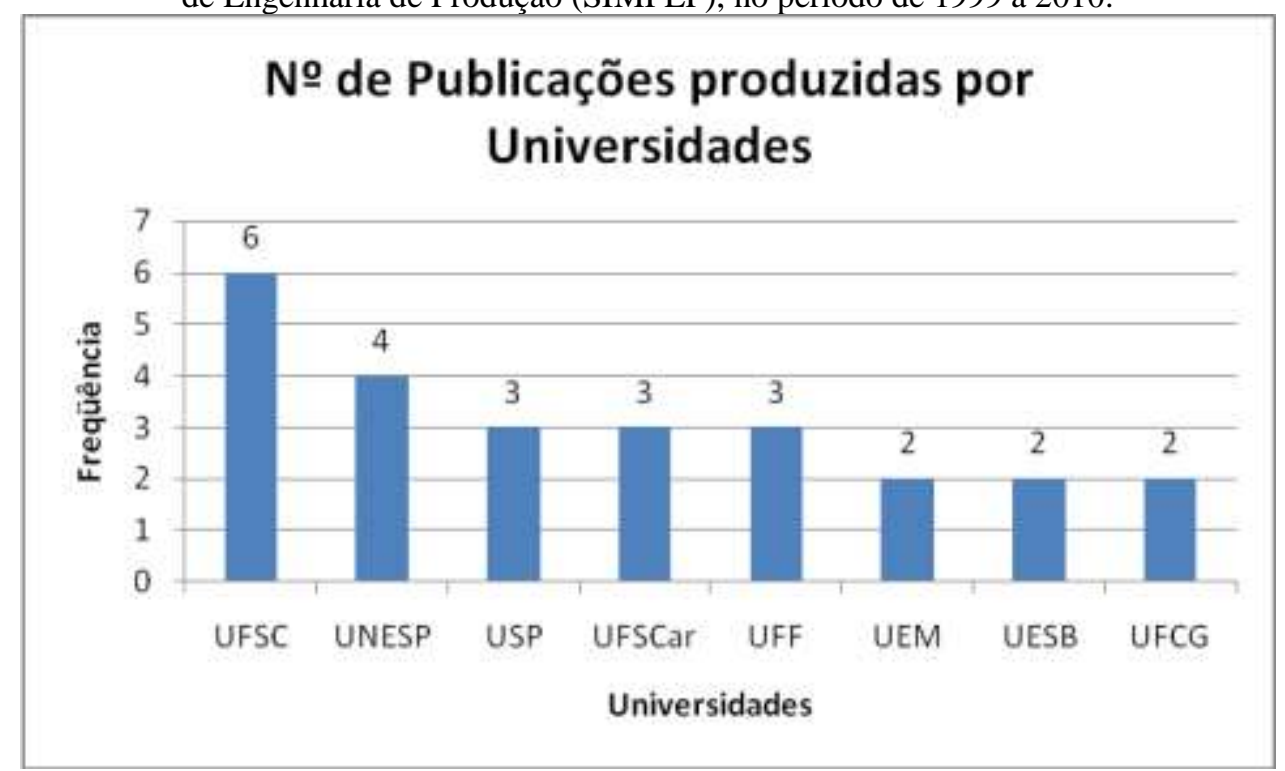

Legenda: UFSC (Universidade Federal de Santa Catarina), UNESP (Universidade Estadual Paulista), USP (Universidade de São Paulo), UFSCar (Universidade Federal de São Carlos), UFF (Universidade Federal Fluminense), UEM (Universidade Estadual de Maringá), UESB (Universidade Estadual do Sudoeste da Bahia) e UFCG (Universidade Federal de Campina Grande).

Fonte: SIMPEP (1999 A 2010)

A Universidade que tem maior número de publicações é a UFSC, com seis artigos, seguida pela UNESP com quatro publicações, a USP, UFSCar e UFF com três artigos cada uma e a UEM, a UESB e a UFCG com dois artigos. Evidencia-se a concentração das publicações nas regiões sudeste e sul, o que foi verificado a partir da freqüência e da localização das instituições de pesquisa identificadas nos artigos, na qual se obteve a distribuição geográfica dos trabalhos sobre ACV no Brasil (Figura 3). Deve se destacar também a região nordeste, dentre as regiões cujas instituições publicaram trabalhos relacionados à ACV no SIMPEP. No entanto, para a Figura 1 não foram consideradas as participações de empresas, porque estas podem estar presentes em diversas regiões, e nem da instituição de Portugal, visto que o objetivo desta pesquisa é identificar estudos sobre ACV no Brasil. 
Figura 3 - Distribuição geográfica das publicações sobre Avaliação de Ciclo de Vida (ACV) no Simpósio de Engenharia de Produção (SIMPEP), no período de 1999 a 2010.

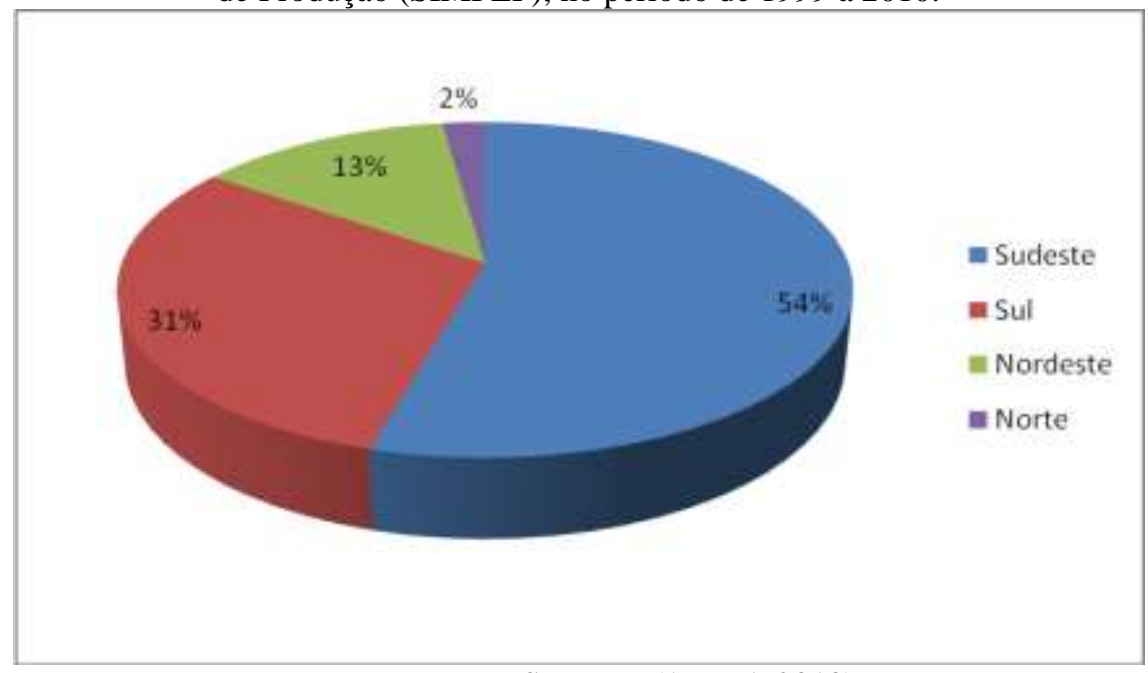

Fonte: SIMPEP (1999 A 2010)

A região sudeste é que representa a maior produção científica na área, com a frequiência de 25 participações (ou 54\%), seguido pela região sul com 14 participações (ou 31\%), de uma freqüência total de 43 participações de universidades brasileiras nos 39 artigos selecionados. A região sudeste tem como influência os programas de pós-graduação e a sua proximidade de centros de tecnologia que se tornaram precursores no emprego da ACV no Brasil, como exemplo o Centro de Tecnologia de Embalagens, do Instituto de Tecnologia de Alimentos, localizado em Campinas, São Paulo. Em relação à região sul, a maior parte destes trabalhos está relacionada com o programa de pós-graduação da UFSC, principalmente, na área de Engenharia de Produção. Recentemente, em 2006, foi formado o Grupo de Pesquisas em Avaliação do Ciclo de Vida da UFSC (2010), concentrando as pesquisas no desenvolvimento e aprimoramento da metodologia de ACV e sua melhor aplicação para as condições ambientais do Brasil.

Entre as regiões foi verificado que as universidades com maior freqüência nos artigos são a UEPA (Universidade do Estado do Pará) com 100\% das participações da região norte, que corresponde a 1 (um) artigo, representando $2 \%$ da produção do país; a UESB (Universidade Estadual do Sudoeste da Bahia) e a UFCG (Universidade Federal de Campina Grande) representam $33 \%$ das participações, cada uma, correspondendo a 2 (dois) artigos de cada, representando $4 \%$ da produção do país; a UFSC com 43\% das participações da região sul, que corresponde a 6 artigos diferentes, representando $14 \%$ do país; e a USP, UNESP e UFSCar correspondendo cada uma a 16\% das participações da região sudeste, com 4 participações em artigos diferentes de cada uma, sendo que estas representando $9 \%$ do país individualmente. Pode-se afirmar que, em relação à divulgação de trabalhos, o SIMPEP tem sido buscado principalmente por instituições das regiões sul e sudeste. Do mesmo modo, se verifica a pouca procura das demais regiões do país, tomando como base a pesquisa feita nos anais do SIMPEP entre os anos de 1999 a 2010. 


\section{2 Áreas de Atuação}

Das 39 publicações encontradas 12 delas concentram seus temas na utilização da ACV como uma ferramenta de gestão ambiental. Há, ainda, a abordagem de assuntos que estão relacionados direta e indiretamente com a gestão ambiental, como o conceito de produção mais limpa (FINGER et al., 2009; GONÇALVES, 2002; ANDRADE; MARINHO; KIPERSTOK, 2001), os sistemas de logística reversa (SCHENINI et al., 2005; RODRIGUES et al., 2005), a concepção de produtos no conceito de ecodesign (BARRA; PASCHOARELLI; RENÓFIO, 2006) ou como metodologia base para projeto de produtos (LUCENTE; NANTES, 2003), as tecnologias limpas (SCHENINI; NEUENFELD; ROSA, 2006), e para os processos de rotulagem ambiental (MACHADO; BIAZIN; GODOY, 1999; GODOY; BIAZIN, 2001; RODRIGUES; ALVES, 2001). De forma geral, outros trabalhos também abordaram o uso da ACV como uma etapa do processo para alcançar a sustentabilidade ambiental (GOUVEIA; MARRA; SILVA, 2004; SANTOS; CARNEIRO; MARTINS, 2005; SAVI; FILHO; SAVI, 2006; CULTRI et al., 2009; FERREIRA et al., 2008; SANTOS et al., 2010a).

Os textos mais específicos, com exceção dos que aplicaram a ACV, abordaram os softwares a serem utilizados para a ACV, realizando comparações entre eles, ressaltando os recursos que cada um oferece (OLIVEIRA; CASTRO; SAFAR, 2007). Alguns abordaram a relação do marketing com a ACV, dissertando como a metodologia pode fornecer dados desde a concepção do produto até o descarte, indicando oportunidade de melhorias contínuas no processo ou no produto, atendendo, assim, a demanda de um mercado com preceitos de responsabilidade sócio-ambiental (CHAVES; MAINIER, 2004; PAVELOSKI; PAVELOSKI, 2006). Ainda foram encontrados dois artigos, sendo um que realizou uma abordagem teórica da aplicação da ACV (SANTOS et al., 2010b) e o outro uma pesquisa bibliográfica sobre a metodologia (WILLERS et al., 2010).

Houve também um texto que apenas mencionou a realização, na França, da ACV de óleos usados, cujo estudo proporcionou informações sobre a melhor disposição final para o produto (TÁVORA; QUELHAS, 2003), sendo contabilizado como citação de aplicação da metodologia. Os artigos restantes são publicações que aplicaram a metodologia de ACV a um estudo de caso e serão discutidos nos próximos tópicos. A figura 4 apresenta uma síntese das áreas abordadas nos trabalhos, citados anteriormente, relacionado com a incidência com que foram citados. 
Figura 4 - O número de artigos selecionados em relação às áreas em que a Avaliação de Ciclo de Vida foi citada, fundamentado na pesquisa documental realizada nos Anais no Simpósio de Engenharia de Produção (SIMPEP) de 1999 a 2010.

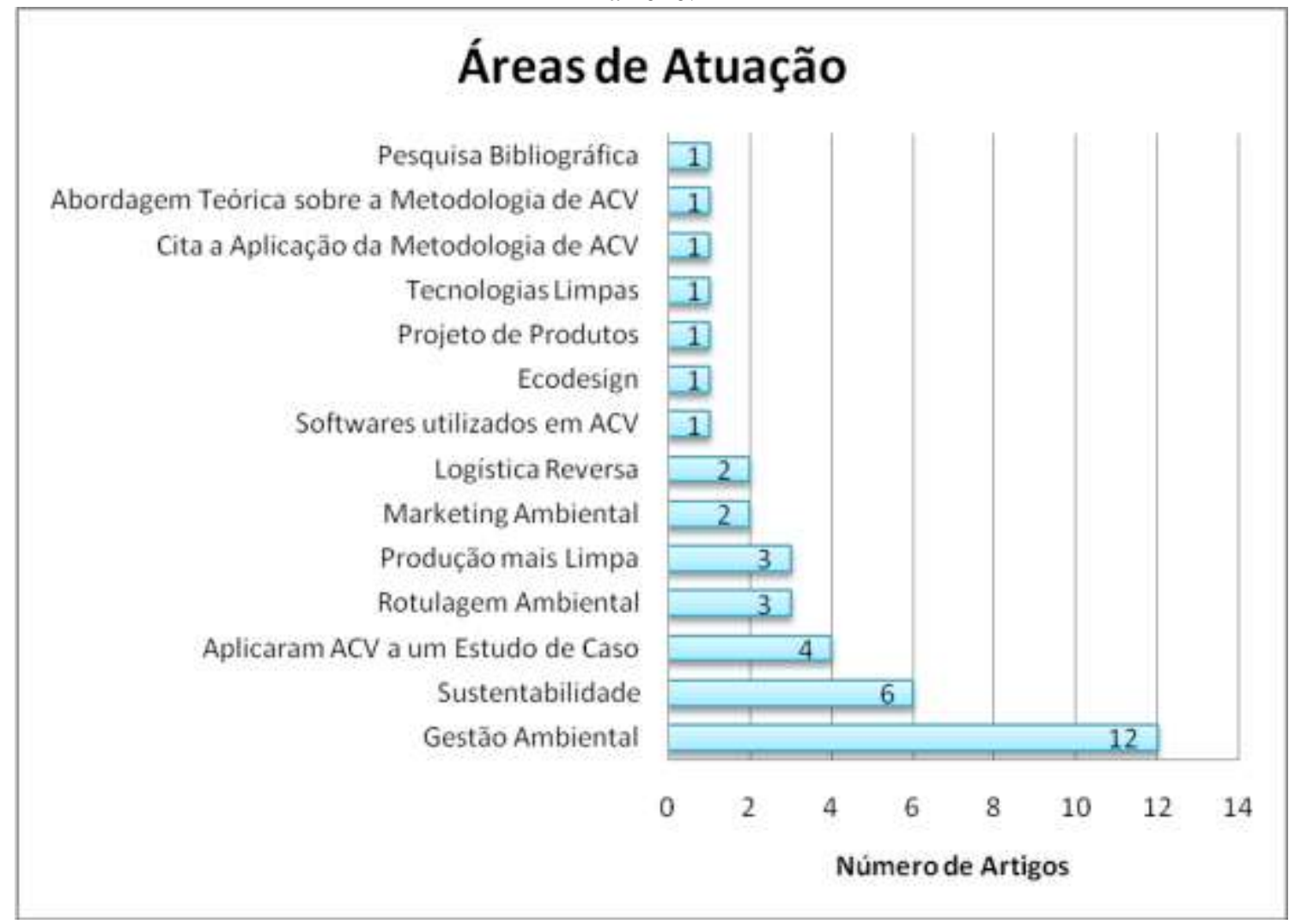

Fonte: SIMPEP (1999 A 2010)

\subsection{Metodologia empregada na aplicação da ACV}

Nesta etapa do trabalho foram selecionados somente os artigos que aplicaram a metodologia de ACV a um estudo de caso, para a realização de uma investigação empírica. Esta investigação procedeu descrevendo as fases desenvolvidas pelos mesmos, com base na metodologia preconizada pela ISO 14040 (2006). Assim, o resultado desta análise foi a identificação das limitações e benefícios das técnicas utilizadas pelos autores, discutidos no tópico posterior.

De acordo com a ISO 14040 (2006) e em seus princípios, a realização da ACV é dividida em fases como: definição de objetivo e escopo, análise de inventário, avaliação de impactos e interpretação de resultados. Deste modo, foram aprofundados os estudos sobre os quatro trabalhos que atenderam ao critério de seleção estabelecido nesta pesquisa.

Nos trabalhos verificados, os estudos de caso de ACV abordaram os seguintes processos e produtos: i) processo de pintura de cabinas de caminhões (GOERGEN; DOKI, 2001); ii) chapas produzidas a partir de resíduos da fabricação de celulose (SANTOS et al., 2007); iii) na comparação do potencial de aquecimento global das pescas artesanal e industrial (SOUZA et al., 2008); e iv) na comparação da utilização de fibras de vidro e juta para obtenção de compósitos pelo processo de Moldagem por Transferência de Resina - Resin Transfer Molding, RTM (SILVA et al., 2009).

$\mathrm{O}$ artigo sobre a ACV no processo de pintura de cabinas de caminhões (GOERGEN; DOKI, 
2001), teve como objetivo a realização de um diagnóstico detalhado do processo, levantar dados que auxiliassem na elaboração dos planos de objetivos e metas exigidos pelo Sistema de Gestão Ambiental (SGA). Para a realização da ACV, foi determinado que o escopo do estudo se delimitasse aos fornecedores primários, ao processo de pintura e aos receptores diretos dos resíduos provenientes do mesmo. A etapa correspondente ao transporte de mercadorias não foi considerada na análise. Adotou-se como unidade funcional a produção média mensal de cabinas durante o período de um ano. O processo foi modelado no software CUMPAN ${ }^{\circledR}$, que também foi utilizado para a avaliação dos impactos ambientais do ciclo de vida, nas seguintes categorias: Eutrofização do Solo e Águas, Geração de Resíduos/Espaço para a Disposição, Redução da Camada de Ozônio, Exaustão de Recursos Naturais, Oxidantes Fotoquímicos, Efeito Estufa (Aquecimento Global) e Chuva Ácida. O trabalho expõe os resultados de maneira quantitativa, indicando através de figuras e gráficos geradas pelo software, os pontos críticos do processo, ou seja, onde ocorre o maior impacto ambiental, no caso a maior contribuição foi para o Efeito Estufa e Consumo de Recursos Naturais.

No artigo sobre a ACV na avaliação chapas produzidas a partir de resíduos da fabricação de celulose (SANTOS et al., 2007), o estudo teve como objetivo avaliar se a disposição final destes resíduos em forma de chapas de vedação é adequada e viável técnica, econômica e ambientalmente. Para a realização da ACV, o escopo do estudo abrangeu todo o ciclo de vida do material, desde a obtenção da matéria-prima, a produção das chapas, o transporte até o consumidor, seu emprego e posterior descarte. Utilizou a metodologia proposta por Mourad, Garcia e Vilhena (2002) e adotou como unidade funcional o $\mathrm{m}^{2}$ da chapa RCB - Resíduo de Celulose e Bambu. Os dados para a composição do inventário do ciclo de vida das chapas foram obtidos através de quantificações práticas em laboratórios e revisão bibliográfica. Já a avaliação do impacto ambiental foi realizada de maneira subjetiva, mas determinou os pontos críticos do ciclo de vida, sendo estes: a distância em que se busca o resíduo de celulose, o processo de secagem do resíduo de celulose, o equipamento conhecido por encoladeira e a resina de uréia-formaldeído.

No artigo sobre a ACV para comparar o potencial de aquecimento global entre as pescas industrial e artesanal (SOUZA et al., 2008), os dados sobre a pesca artesanal foram obtidos por meio de entrevistas junto aos pescadores locais, como também através dos resultados estruturados em relatórios do projeto Ressurgência, da Universidade Federal do Rio de Janeiro. Já os dados da pesca industrial foram coletados numa empresa do setor. A análise dos dados foi feita utilizando-se o programa computacional Umberto ${ }^{\circledR}$, adotando como unidade funcional uma tonelada de peixe da espécie "pargo", in natura. O sistema abrangeu desde a pesca, industrialização, transporte, distribuição até o descarte do produto. Durante a análise e cálculo de emissões de gases, foram considerados como potenciais de aquecimento global: o dióxido de carbono $\left(\mathrm{CO}_{2}\right)$, o metano $\left(\mathrm{CH}_{4}\right)$ e o monóxido de dinitrogênio $\left(\mathrm{N}_{2} \mathrm{O}\right)$. Estes foram transformados em quantidade de dióxio de 
carbono equivalente para a avaliação do impacto ambiental, utilizando os fatores de potencial de aquecimento global provenientes da tabela do IPCC (Intergovernmental Painel on Climate Change). $\mathrm{O}$ artigo demonstra, de maneira quantitativa, que a pesca industrial tem maior impacto no aquecimento global.

Por último, o artigo sobre a ACV de materiais compósitos produzidos pelo processo RTM Resin Transfer Molding, (SILVA et al., 2009), teve como objetivo avaliar os impactos ambientais relacionados com o processo RTM para produzir materiais compósitos feitos de fibras de vidro e juta, empregando a metodologia da ACV. O trabalho utilizou o programa computacional SIMAPRO $^{\circledR} 7.1$ para análise de dados e avaliação dos impactos ambientais. A unidade funcional considerada foi uma placa de material compósito. Estabelecida a fronteira apenas na fase de produção dos materiais compósitos via RTM, concluiu-se que todos os impactos ambientais estão relacionados à produção de energia elétrica, por esta ser a única entrada no sistema, e cujo impacto mais evidente é o smog de verão. O resultado da comparação da ACV da fibra de vidro e de juta, é que a fibra de vidro, na fase de produção, apresenta menor impacto ambiental, devido ao menor consumo de energia para a composição deste material na fase de injeção.

As principais características dos quatros trabalhos encontrados podem ser visualizados na Tabela 1. Estas características estão relacionadas aos objetivos, escopo, unidade funcional, metodologia, ferramentas ou softwares para a avaliação de ciclo de vida (ACV) ou avaliação de impacto ambiental (AIA) e os resultados obtidos pelo trabalho.

Tabela 1 - Síntese dos artigos s que aplicaram a Análise de Ciclo de Vida á um estudo de caso no Simpósio de Engenharia de Produção (SIMPEP), no período de 1999 até 2010

\begin{tabular}{|c|c|c|c|c|c|}
\hline Autores & Objetivos & Escopo & $\begin{array}{c}\text { Unidade } \\
\text { Funcional }\end{array}$ & $\begin{array}{c}\text { Metodologia, } \\
\text { ferramentas } \\
\text { ou softwares } \\
\text { para a ACV e } \\
\text { AIA }\end{array}$ & Resultados \\
\hline $\begin{array}{c}\text { Goergen } \\
\text { e Doki, } \\
(2001)\end{array}$ & $\begin{array}{c}\text { Realização de um } \\
\text { diagnóstico } \\
\text { detalhado do } \\
\text { processo, levantar } \\
\text { dados confiáveis que } \\
\text { auxiliassem na } \\
\text { elaboração dos } \\
\text { planos de objetivos e } \\
\text { metas exigidos pelo } \\
\text { SGA }\end{array}$ & $\begin{array}{l}\text { Delimitou-se aos } \\
\text { fornecedores } \\
\text { primários, ao } \\
\text { processo de pintura } \\
\text { em si e aos } \\
\text { receptores diretos } \\
\text { dos resíduos } \\
\text { provenientes do } \\
\text { mesmo. }\end{array}$ & $\begin{array}{l}\text { Produção } \\
\text { média } \\
\text { mensal de } \\
\text { cabinas } \\
\text { durante o } \\
\text { período de } \\
\text { um ano }\end{array}$ & $\begin{array}{c}\text { Software: } \\
\text { CUMPAN }^{\circledR}\end{array}$ & $\begin{array}{l}\text { Figuras e gráficos } \\
\text { gerados pelo } \\
\text { software indicam os } \\
\text { pontos críticos do } \\
\text { processo } \\
\text { relacionados com as } \\
\text { categorias de } \\
\text { impactos } \\
\text { selecionadas }\end{array}$ \\
\hline
\end{tabular}




\begin{tabular}{|c|c|c|c|c|c|}
\hline $\begin{array}{l}\text { Santos et } \\
\text { al. }, \\
(2007)\end{array}$ & $\begin{array}{l}\text { Avaliar se a } \\
\text { disposição final dos } \\
\text { resíduos de celulose } \\
\text { em forma de chapas } \\
\text { de vedação é } \\
\text { adequada e viável } \\
\text { técnica, econômica e } \\
\text { ambientalmente }\end{array}$ & $\begin{array}{l}\text { Desde a obtenção } \\
\text { da matéria-prima, a } \\
\text { produção das } \\
\text { chapas, o transporte } \\
\text { até o consumidor, } \\
\text { seu emprego e } \\
\text { posterior descarte }\end{array}$ & $\begin{array}{l}\mathrm{m}^{2} \text { da chapa } \\
\text { RCB - } \\
\text { Resíduo de } \\
\text { Celulose e } \\
\text { Bambu }\end{array}$ & $\begin{array}{l}\text { Metodologia } \\
\text { proposta por } \\
\text { Mourad, } \\
\text { Garcia e } \\
\text { Vilhena (2002) } \\
\text { AIA: realizado } \\
\text { de maneira } \\
\text { subjetiva }\end{array}$ & $\begin{array}{l}\text { Determinou os } \\
\text { pontos críticos do } \\
\text { ciclo de vida, sendo } \\
\text { estes: a distância em } \\
\text { que se busca o } \\
\text { resíduo de celulose, } \\
\text { o processo de } \\
\text { secagem do resíduo } \\
\text { de celulose, o } \\
\text { equipamento } \\
\text { conhecido por } \\
\text { encoladeira e a } \\
\text { resina de uréia- } \\
\text { formaldeído }\end{array}$ \\
\hline $\begin{array}{l}\text { Souza et } \\
\text { al., } \\
(2008)\end{array}$ & $\begin{array}{c}\text { Comparar o } \\
\text { potencial de } \\
\text { aquecimento global } \\
\text { entre as pescas } \\
\text { industrial e artesanal }\end{array}$ & $\begin{array}{c}\text { Abrangeu desde a } \\
\text { pesca, } \\
\text { industrialização, } \\
\text { transporte, } \\
\text { distribuição até o } \\
\text { descarte do produto }\end{array}$ & $\begin{array}{l}1 \text { (uma) } \\
\text { tonelada de } \\
\text { peixe da } \\
\text { espécie } \\
\text { "pargo", in } \\
\text { natura }\end{array}$ & $\begin{array}{l}\text { Software: } \\
\text { Umberto }^{\circledR}\end{array}$ & $\begin{array}{l}\text { A pesca industrial } \\
\text { tem maior impacto } \\
\text { no aquecimento } \\
\text { global }\end{array}$ \\
\hline $\begin{array}{l}\text { Silva et } \\
\text { al., } \\
(2009)\end{array}$ & $\begin{array}{l}\text { Avaliar os impactos } \\
\text { ambientais } \\
\text { relacionados com o } \\
\text { processo RTM para } \\
\text { produzir materiais } \\
\text { compósitos feitos de } \\
\text { fibras de vidro e juta, } \\
\text { empregando a } \\
\text { metodologia da ACV }\end{array}$ & $\begin{array}{l}\text { Fase de produção } \\
\text { dos materiais } \\
\text { compósitos via } \\
\text { RTM }\end{array}$ & $\begin{array}{l}1 \text { (uma) } \\
\text { placa de } \\
\text { material } \\
\text { compósito }\end{array}$ & $\begin{array}{c}\text { Software: } \\
\text { SIMAPRO }^{\circledR} \\
7.1\end{array}$ & $\begin{array}{l}\text { A fibra de vidro, na } \\
\text { fase de produção, } \\
\text { apresenta menor } \\
\text { impacto ambiental, } \\
\text { devido ao menor } \\
\text { consumo de energia } \\
\text { para a composição } \\
\text { deste material na } \\
\text { fase de injeção }\end{array}$ \\
\hline
\end{tabular}

Fonte: SIMPEP (1999 A 2010)

\subsection{Benefícios e limitações identificadas na metodologia de ACV}

Tendo em vista que qualquer metodologia possui benefícios e limitações, pressupõe-se que a compreensão destes é importante para estabelecer a abrangência dos estudos que o aplicam. Assim, este tópico aborda os benefícios e limitações relatados pelos autores dos 4 artigos que aplicaram a metodologia de ACV a um estudo de caso.

Em relação aos benefícios, Santos et al. (2007) relatam que por meio da ACV, foi possível perceber claramente os pontos do processo onde existia um grande desperdício de materiais, energia e emissão desnecessária de poluentes, tornando o processo muito oneroso e inviável financeiramente. A ACV apresentou-se como uma ferramenta essencial para promover melhorias no produto dos pontos de vista técnico, econômico e ambiental.

Análogo a Santos et al. (2007), Goergen e Doki (2001) e Souza et al. (2008) relatam que a aplicação da metodologia de ACV mostrou-se eficaz e bastante prática, pois permite identificar pontos críticos dentro dos processos, os quais deverão ser alvos prioritários dos planos de ação, além de fornecer dados em unidades passíveis de confrontação com parâmetros citados em legislação, acordos internacionais ou definições das próprias empresas, para averiguação de conformidade ou não. Além da identificação e avaliação dos aspectos ambientais, esta é uma 
tecnologia capaz de oferecer uma visão mais ampla de toda cadeia produtiva, facilitando assim sua gestão.

Já Silva et al. (2009) foi mais específico, e disse que ao utilizar a metodologia de ACV foi possível identificar os impactos relacionados à fase de produção. No entanto, ressaltou que mais esforços devem concentrar-se para outras fases, principalmente na fase de eliminação, que, segundo Joshi (2004) apud Silva et al. (2009) é muito poluente em materiais compósitos.

Agora, em relação às limitações da metodologia ACV, Souza et al. (2008) relatam que os cálculos das emissões de dióxido de carbono, metano e monóxido de nitrogênio, foram feitos com base em indicadores desenvolvidos por estudos europeus tirados da base de dados do programa computacional utilizado. Este é um problema recorrente nos trabalhos de ACV no Brasil, o que reflete a necessidade de um banco de dados brasileiro. Talvez este seja um dos motivos que tem contribuído para o limitado avanço dos estudos de ACV no país.

Por fim, identificou-se que a etapa de Avaliação de Impactos é, sem dúvida, a mais difícil da ACV no sentido de se atingir um consenso científico internacional sobre a metodologia mais adequada. Não há uma técnica que, sozinha, forneça uma completa avaliação ambiental. Uma avaliação pode ter que atender a diferentes necessidades nos processos de tomada de decisão e diferentes técnicas poderão ser necessárias para prover informações. Há que se considerar as limitações do conhecimento científico no estabelecimento dos impactos potenciais, aspectos como subjetividade nas análises ou diferenças entre indicadores e impactos. Assim, o processo conceitual e métodos de avaliação de impactos da ACV são recentes e continuam em desenvolvimento. (GOERGEN; DOKI, 2001; SANTOS et al., 2007).

\section{Considerações finais}

A pesquisa identificou 39 artigos, dos quais 35 se restringiram a uma abordagem conceitual da ACV, sem uma aplicação efetiva da metodologia. Percebeu-se, porém, que na maioria destes trabalhos a ACV poderia ser plenamente aplicada, revelando assim potenciais campos para a utilização da metodologia. Áreas como construção civil, agroindústria, logística, etc; são passíveis de aplicação da ACV com perspectiva de resultados promissores acerca dos impactos ambientais. Observa-se, ainda, que dos 35 artigos, apenas 18 citaram ACV em seu contexto sem definir a metodologia, enquanto os outros 17 mencionaram e definiram a metodologia.

Avaliando os 39 trabalhos, verificou-se a participação de 30 diferentes instituições pesquisa, sendo 27 destas universidades brasileiras, e que relacionado com a freqüência e localização destas instituições, foi possível identificar como as principais universidades, que abordam o tema ACV (UFSC, UNESP, USP, UFSCar, UFF, UEM, UESB e UFCG), e as regiões sudeste e sul como maior produção científica na área, e o recente crescimento da participação da região nordeste, que 
de um total de seis participações, as últimas quatro foram na edição de 2010.

Já os trabalhos da região norte e sul são predominantes da UEPA e da UFSC que, percentualmente são as instituições que possuem maior freqüência de participação nas publicações para uma região, com $100 \%$ e $43 \%$ dos trabalhos produzidos, respectivamente. Em seguida tem-se a UESB, UFCG, cada uma, com 33\% dos trabalhos da região nordeste, e a UFSCar, UNESP e USP, cada uma, com 16\% dos trabalhos da região sul. Ressalta-se, ainda, a ausência de participação das instituições da região centro-oeste e, também, o baixo número de participação das instituições da região norte e nordeste, o que pode caracterizar, a princípio, a pouca procura pelo SIMPEP para a divulgação dos trabalhos pelas instituições destas regiões.

A pesquisa realizada verificou um número considerável de trabalhos que abordam o tema, contudo, houve um baixo número destes, apenas 10,3\%, que aplicaram efetivamente a metodologia da ACV, tendo como base os anais do SIMPEP de 1999 a 2010. Ressalta-se que dos quatro trabalhos citados neste estudo, três foram publicados nas últimas edições do evento (2007, 2008 e 2009), refletindo a recente tendência de trabalhos mais consistentes sobre ACV no SIMPEP.

Também, constata-se nos trabalhos que aplicaram a ACV a um estudo de caso, que esta é uma metodologia que identifica os pontos críticos do processo, fornece subsídios necessários à elaboração de planos de ação e acompanhamento da melhoria contínua almejada pelo Sistema de Gestão Ambiental, e ainda identifica e quantifica os impactos ambientais ao longo do ciclo de vida do produto ou processo.

A ACV é uma ferramenta com grande potencial de aplicação na avaliação de impactos ambientais. Esta por sua vez é a etapa mais difícil da ACV no sentido de se atingir um consenso científico internacional sobre a metodologia mais adequada. Há que se considerar as limitações do conhecimento científico no estabelecimento dos impactos potenciais, aspectos como subjetividade nas análises ou diferenças entre indicadores e impactos. Assim, o processo conceitual e métodos de avaliação de impactos da ACV são recentes e continuam em desenvolvimento. Igualmente, a utilização de indicadores baseados em estudo europeus é um problema recorrente nos trabalhos de ACV no Brasil, o que reflete a necessidade de um banco de dados brasileiro. Talvez este seja um dos motivos que tem limitado o avanço dos estudos de ACV no país.

Deste modo, sugere-se a difusão dessa metodologia através da abordagem direta do tema pelo SIMPEP em seus fóruns de discussão, por meio da promoção de palestras e/ou mini-cursos, ou incentivar o tema ACV em suas áreas temáticas para submissão de artigos, de modo à melhor divulgar e propagar a metodologia ACV para profissionais e pesquisadores das diversas regiões do país.

\section{Abstract}


In recent years studies about the Life Cycle Assessment (LCA) methodology has been growing in Brazil and yet can be noted in the main study/research centers. Despite recent, it is necessary to know about LCA developments in the country, through the verification of scientific literature produced. So in this work was performed a bibliography research about LCA in the proceedings of the Brazilian scientific conference, Simpósio de Engenharia de Produção SIMPEP, to select articles that addressed its thematic. Following, an exploratory and descriptive research in the previous selected works was performed by identifying the research institutions, issues related to LCA and case studies that applied the methodology. Finally, were analyzed only the studies that effectively used the LCA, resulting relevant information that were compared in this small group. The study considered 39 articles, where were identified 30 different research institutions, whose 27 are universities. The universities with more frequency in the articles were UFSC, UNESP, USP, UFSCar, UFF, UEM, UESB and UFCG. It is noted that from the 39 articles selected, 35 were restricted to a conceptual approach of the LCA, without an effective application of the methodology, whose references were mostly related to the Environmental Management and Sustainability. Thus, it was found four articles (10.3\%), which effectively applied the LCA methodology. From this works, three were published in the recent events editions (2007, 2008 and 2009), reflecting the recent trend of more consistent work on LCA in the SIMPEP.

Key-words: Life Cycle Assessment, ISO 14040; SIMPEP.

\section{Referências}

ALVES, C.; FERRÃO, P. M. C.; SILVA, A. J.; REIS, L. G.; FREITAS, M.; RODRIGUES, L. B.; ALVES, D. E. Ecodesign of automotive components making use of natural jute fiber composites. Journal of Cleaner Production, $v$. 18 , p. 313-327, 2010.

\section{cross ref}

ANDRADE, J. C. S.; MARINHO, M. M. O.; KIPERSTOK, A. Política Ambiental focada na Produção Limpa: elementos para discussão com os setores produtivos. In: Simpósio de Engenharia de Produção, VIII, 2001 , Bauru. Anais... Simpósio de Engenharia de Produção, Bauru: UNESP, 2001.

ASSOCIAÇÃO BRASILEIRA DE NORMAS TÉCNICAS - ABNT. NBR ISO 14040: Gestão ambiental - Avaliação do ciclo de vida - Princípios e estrutura. Rio de Janeiro, 2001. 10 p.

BARRA, B. N.; PASCHOARELLI, L. C.; RENÓFIO, A. O ecodesign como ferramenta de auxílio na gestão de resíduos de construção e demolição (RCD). In: Simpósio de Engenharia de Produção, XIII, 2006, Bauru. Anais... Simpósio de Engenharia de Produção, Bauru: UNESP, 2006.

BORGES, F. H.; TACHIBANA, W. K. O quadro evolutivo do ambientalismo e os impactos no ambiente dosnegócios. In: Simpósio de Engenharia de Produção, XII, 2005, Bauru. Anais... Simpósio de Engenharia de Produção, Bauru: UNESP, 2005.

CHAVES, L. A.O.; MAINIER, F. B. Análise do Ciclo de Vida de Produto e seus Impactos no Mercado: Uso de Produtos Ecológicos. In: Simpósio de Engenharia de Produção, XI, 2004, Bauru. Anais... Simpósio de Engenharia de Produção, Bauru: UNESP, 2004.

CHEHEBE, J. R. Análise do ciclo de vida de produtos: Ferramenta gerencial da ISO 14000. Rio de Janeiro: Qualitymark, 1997.

CULTRI, C.N.; ALVES, V.C.; OMETTO, A.R.; RENOFIO, A. Responsabilidade social empresarial: panorama dos instrumentos de avaliação e gestão. In: Simpósio de Engenharia de Produção, XVI, 2009, Bauru. Anais... Simpósio de Engenharia de Produção, Bauru: UNESP, 2009.

FERREIRA, A. R.; JOÃO, D. M.; CARVALHO, M. N. M.; AITA, J. A. A.; JÚNIOR, E. G. M. Utilização de resíduos na fabricação de chapas MDF (Medium-density fiber board). In: Simpósio de Engenharia de Produção, XV, 2008, Bauru. Anais... Simpósio de Engenharia de Produção, Bauru: UNESP, 2008.

FIGUEIRÊDO, M. C. B.; MOTA, S.; RODRIGUES, G. S.; CALDEIRA-PIRES, A.; ROSA, M. F. Metodologia de avaliação dos impactos ambientais de inovações tecnológicas agroindustriais, com base no ciclo de vida. In: Conferência Internacional Ciclo de Vida, 2007, São Paulo. Anais... Conferência Internacional Ciclo de Vida, São Paulo: 2007.

FINGER, L.; NETO, L. M.; CAVALCANTI, M. M.; HARTMANN, A.; COSTA, A. M. Análise do Sistema de Gestão Ambiental do Laboratório de Camarões Marinhos da Universidade Federal de Santa Catarina. In: Simpósio de Engenharia de Produção, XVI, 2009, Bauru. Anais... Simpósio de Engenharia de Produção, Bauru: UNESP, 2009.

GIL, Antônio C. Como elaborar projetos de pesquisa. São Paulo: Atlas: 1995.

GODOY, A. M. G.; BIAZIN, C. C. Reestruturação Produtiva e Barreiras: a Rotulagem Ambiental. In: Simpósio de Engenharia de Produção, VIII, 2001, Bauru. Anais... Simpósio de Engenharia de Produção, Bauru: UNESP, 2001. 
GOERGEN, L. R.; DOKI, C. Análise do ciclo de vida - ferramenta para garantia da melhoria contínua em processos produtivos. In: Simpósio de Engenharia de Produção, VIII, 2001, Bauru. Anais... Simpósio de Engenharia de Produção, Bauru: UNESP, 2001.

GONÇALVES, L. C. Produção Mais Limpa como Instrumento de Gestão Ambiental para a Hotelaria Nacional. In: Simpósio de Engenharia de Produção, IX, 2002, Bauru. Anais... Simpósio de Engenharia de Produção, Bauru: UNESP, 2002.

GOUVEIA, A. B. O.; MARRA, F. A.; SILVA, C. E. S. Análise crítica de um produto sob o aspecto da sustentabilidade. In: Simpósio de Engenharia de Produção, XI, 2004, Bauru. Anais... Simpósio de Engenharia de Produção, Bauru: UNESP, 2004.

GUINÉE, J. Development of a methodology for the Environmental Life-Cycle Assessment of Products (with a case study on margarines). Ph. D. thesis. Leiden University, Leiden, 1995. Disponível em: <https://openaccess.leidenuniv.nl/handle/1887/8052>. Acesso em: jan. 2011.

HUNT, R.; Franklin, E. (1996). LCA - How it Came About. Personal Reflections on the Origin and the Development of LCA in the USA. Int. J. LCA, vol. 1 (1) 4-7. Landsberg, Germany: Ecomed.

\section{cross $r$ ref}

INTERGOVERNMENTAL PAINEL ON CLIMATE CHANGE. Third Assessment Report - Climate Change 2001: $\begin{array}{lllll}\text { Working Group } & \text { I. } & \text { [S.I.]: } & \text { GRID-Arendal, } & \text { Disponível }\end{array}$ <http://www.grida.no/publications/other/ipcc_tar/?src=/climate/ipcc_tar/wg1/248.htm>. Acesso em: abr. 2010.

INTERNATIONAL ORGANIZATION FOR STANDARDIZATION (2000). ISO/TR 14047: Life Cycle Impact Assessment. Examples of Application of ISO 14042.

INTERNATIONAL ORGANIZATION FOR STANDARDIZATION (2000). ISO/TR 14049: Life Cycle Assessment. Examples of Application of SO 14041 for goal and scope definition and inventory analysis.

INTERNATIONAL ORGANIZATION FOR STANDARDIZATION (2001). ISO/TS 14048: Life Cycle Assessment. Data Documentation Format.

INTERNATIONAL ORGANIZATION FOR STANDARDIZATION (2006). ISO 14040: Life Cycle Assessment. Principles and Framework.

INTERNATIONAL ORGANIZATION FOR STANDARDIZATION (2006). ISO 14044: Life Cycle Assessment. Requirements and Guidelines.

JOSHI, S. V.; DRZAL, L. T.; MOHANTY, A. K.; ARORA, S. Are natural fiber composites environmentally superior to glass fiber reinforced composites? Composites Part A: Applied Science and Manufacturing, v. 35, n. 3, p. 371-376, 2004.

\section{cross $r$ ref}

LIMA, A. M. F. Avaliação do Ciclo de Vida no Brasil: inserção e perspectivas. Dissertação de Mestrado: UFBA Escola Politécnica, Salvador, 2007.

LUCENTE, A. R.; NANTES, J. F. D. Avaliação Ambiental no Processo Produtivo: Um Estudo no Setor de Produtos Veterinários. In: Simpósio de Engenharia de Produção, X, 2003, Bauru. Anais... Simpósio de Engenharia de Produção, Bauru: UNESP, 2003.

MACHADO, H. V.; BIAZIN, C. C.; GODOY, A. M. G. Rotulagem Ambiental para pequenas empresas do setor moveleiro: dilemas e perspectivas. In: Simpósio de Engenharia de Produção, VI, 1999, Bauru. Anais... Simpósio de Engenharia de Produção, Bauru: UNESP, 1999.

MOURAD, A.L.; GARCIA, E.E.C.; VILHENA, A. Avaliação do Ciclo de Vida: Princípios e Aplicações. Campinas: CETEA/CEMPRE, 92p, 2002.

OLIVEIRA, P. M.; CASTRO, C. G.; SAFAR, G. H. Softwares utilizados na Avaliação do ciclo de Vida (ACV) - Uma abordagem analítica. In: Simpósio de Engenharia de Produção, XIV, 2007, Bauru. Anais... Simpósio de Engenharia de Produção, Bauru: UNESP, 2007.

PAVELOSKI, A.; PAVELOSKI, E. M. Avaliação do ciclo de vida (ACV) como instrumento para ações de marketing. In: Simpósio de Engenharia de Produção, XIII, 2006, Bauru. Anais... Simpósio de Engenharia de Produção, Bauru: UNESP, 2006.

PEGORARO, L.A. Desenvolvimento de fatores de caracterização para toxicidade humana em avaliação do impacto do ciclo de vida no Brasil. Dissertação de Mestrado: UTFPR - Programa de Pós-Graduação em Engenharia Mecânica e de Materiais, Curitiba, 2008. 
RIZZO, M. R.; OLIVEIRA, E. C. A questão ambiental no contexto das organizações: responsabilidade socioambiental ou uma forma de diferenciação para o mercado? In: Seminário Temático Centralidade e Fronteiras das Empresas no Século XXI, 2007, São Carlos/SP. Seminário Temático Centralidade e Fronteiras das Empresas no Século XXI. São Carlos/SP: Núcleo de Estudos em Sociologia Econômica e das Finanças (NESEFI) - PPGEP/UFSCar/SP, 2008. p. 1-18.

RODRIGUES, A. M.; RODRIGUES, I. C.; REBELATO, M. G.; SCHUINDT, A. A logística reversa como instrumento de geração de renda e inclusão social: o caso de uma empresa produtora de pneus remoldados. In: Simpósio de Engenharia de Produção, XII, 2005, Bauru. Anais... Simpósio de Engenharia de Produção, Bauru: UNESP, 2005.

RODRIGUES, I. C.; ALVES, F. J. C. Certificações de Caráter Ambiental nos Sistemas Produtivos Agroindustriais. In: Simpósio de Engenharia de Produção, VIII, 2001, Bauru. Anais... Simpósio de Engenharia de Produção, Bauru: UNESP, 2001.

SANTOS, J. G.; ABREU, T. A.; VICTOR, D. L.; RAMALHO, A. M. C. Sustentabilidade empresarial a partir da adoção da produção mais limpa: um estudo de caso em uma empresa do setor moveleiro. In: SIMPÓSIO DE ENGENHARIA DE PRODUÇÃ̃, XVII, 2010, Bauru. Anais... Bauru: UNESP, 2010a.

SANTOS, J. G.; SILVA, S. S. F.; RAMALHO, A. M. C.; NOGUEIRA, V. B. M. Avaliação do Ciclo de Vida como instrumento de Gestão Ambiental: uma abordagem teórica. In: SIMPÓSIO DE ENGENHARIA DE PRODUÇÃO, XVII, 2010, Bauru. Anais... Bauru: UNESP, 2010b.

SANTOS, L. M. M. Avaliação ambiental de processos industriais. 2 ed. São Paulo: Signus Editora, 2006. 130p.

SANTOS, M. F. N.; BATtiSTElle, R. A. G.; MiYAZATO, T.; FREITAS, P. N. P. A Análise do Ciclo de Vida na avaliação de chapas produzidas a partir de resíduos da fabricação de celulose. In: Simpósio de Engenharia de Produção, XIV, 2007, Bauru. Anais... Simpósio de Engenharia de Produção, Bauru: UNESP, 2007.

SANTOS, N. S. S.; CARNEIRO, S. C. P.; MARTINS, H. S. Utilização do Trançado de Palha como estratégia para o desenvolvimento sustentável do setor moveleiro. In: Simpósio de Engenharia de Produção, XII, 2005, Bauru. Anais... Simpósio de Engenharia de Produção, Bauru: UNESP, 2005.

SAVI, A. F.; FILHO, E. V. G.; SAVI, E. M. S. Engenharia apoiando o desenvolvimento sustentável. In: Simpósio de Engenharia de Produção, XIII, 2006, Bauru. Anais... Simpósio de Engenharia de Produção, Bauru: UNESP, 2006.

SCHENINI, P. C.; NEUENFELD, D. R.; ROSA, A. L. M. O gerenciamento de riscos no transporte de produtos perigosos. In: Simpósio de Engenharia de Produção, XIII, 2006, Bauru. Anais... Simpósio de Engenharia de Produção, Bauru: UNESP, 2006.

SCHENINI, P. C.; NEUNFELD, D. R.; MULLER, G. S.; RENSI, F. Logística reversa: um estudo de caso. In: Simpósio de Engenharia de Produção, VIII, 2005, Bauru. Anais... Simpósio de Engenharia de Produção, Bauru: UNESP, 2005.

SIlvA, C. A.; RODRIGUES, L. B.; ESTAREGUE, D.; FERRÃO, P. M. C.; FREITAS, M. Using Life Cycle Assessment to identify the environmental impacts of composite materials produced via RTM process. In: Simpósio de Engenharia de Produção, XVI, 2009, Bauru. Anais... Simpósio de Engenharia de Produção, Bauru: UNESP, 2009.

SIMAPRO. SimaPro LCA software. Disponível em: <http://www.pre.nl/simapro/>; Acesso em: abr. 2010.

SIMPÓSIO DE ENGENHARIA DE PRODUÇÃO, 1999 a 2009, Bauru. Anais eletrônicos... Bauru: UNESP, 2010. Disponível em: <http://www.simpep.feb.unesp.br/anais.php>; Acess em: Jan. 2010.

SOUZA, D. P.; MENDONÇA, F. M.; VAlle, R. A. B; CARNEIRO, A. M.; NUNES, K. R. A. A Aplicação da Metodologia de Avaliação do Ciclo de Vida (ACV) para comparar o potencial de Aquecimento Global entre as pescas industrial e artesanal: um estudo nas áreas de Cabo Frio e Arraial do Cabo. In: Simpósio de Engenharia de Produção, XV, 2008, Bauru. Anais... Simpósio de Engenharia de Produção, Bauru: UNESP, 2008.

TÁVORA, S. P.; QUELHAS, O. L. G. Óleos Lubrificantes usados - Evolução das Responsabilidades pela Coleta/ Destinação e alternativas para aplicações. In: Simpósio de Engenharia de Produção, X, 2003, Bauru. Anais... Simpósio de Engenharia de Produção, Bauru: UNESP, 2003.

UMBERTO. Software de gestão ambiental e análise de fluxos de materiais e energia. Disponível em: <http://www.umberto.de/en/home/language/portuguese/>. Acesso em: abr. 2010.

Universidade Federal de Santa Catarina. Grupo de Pesquisas em Avaliação do Ciclo de Vida. Disponível em: <http://www.ciclodevida.ufsc.br/acv/Main.php?do=adminAction\&action=exibirSubMenu\&idSubMenu=27>. Acesso em: mar. 2011.

VIGON, B. W.; TOLLE, D. A.; CORNABY, B. W.; LATHAM, H. C.; HARRISON, C. L.; BOGUSKI, T. L.; HUNT, R. G.; SLLERS, J. D. Life-Cycle Assessment: Inventory Guidelines and Principles. United States Environmental Protection Agency. 1993.

WILlERS, C. D.; RODRIGUES, L. B.; SIlvA, N. L.; SILVA, C. A. Avaliação do Ciclo de Vida: Uma Pesquisa 
Bibliográfica nos Anais do SIMPEP. In: Simpósio de Engenharia de Produção, XVII, 2010, Bauru. Anais... Simpósio de Engenharia de Produção, Bauru: UNESP, 2010.

\section{Dados dos autores:}

\section{Nome completo: Camila Daniele Willers}

Filiação institucional: Universidade Estadual do Sudoeste da Bahia

Função ou cargo ocupado: Estudante de Graduação / Bolsista de Iniciação Científica

Endereço completo para correspondência (bairro, cidade, estado, país e CEP): Pç. Primavera, 40 Primavera. Itapetinga, Bahia. 45700-000

Telefones para contato: (77)8815-6376

e-mail: rodrigueslb@gmail.com

\section{Nome completo: Luciano Brito Rodrigues}

Filiação institucional: Universidade Estadual do Sudoeste da Bahia

Departamento: Tecnologia Rural e Animal

Função ou cargo ocupado: Professor Adjunto

Endereço completo para correspondência (bairro, cidade, estado, país e CEP): Pç. Primavera, 40 Primavera. Itapetinga, Bahia. 45700-000

Telefones para contato: (77) 3261-8651 / (77) 3261-8611 / (77) 8804-9974

e-mail: rodrigueslb@gmail.com

Enviado em: 12/06/2012

Aprovado em: 22/03/2012 